\title{
Suppression of Reperfusion Injury in Rat Skeletal Muscle by Antioxidative Enzymes ${ }^{\mathrm{a}}$
}

\author{
Atsushi Yamamoto, ${ }^{1}$ Hitoshi TaKenaKa, ${ }^{2, b}$ Koh-ichiroh Shibata, ${ }^{1}$ \\ Toshio ONITSUKA, ${ }^{1}$ Yasunori KogA, ${ }^{2, c}$ \\ and Minoru HAMADA, ${ }^{2, d, *}$ \\ ${ }^{1}$ Second Department of Surgery and ${ }^{2}$ Department of Hygiene, \\ Miyazaki Medical College, Miyazaki 889-16, Japan
}

(Received May 15, 1997)

\begin{abstract}
Summary Prolonged skeletal muscle ischemia has been reported in acute arterial occlusion and crush syndrome. It often induces progressive edema and necrosis and occasionally life-threatening systemic complications, such as hypotension, hyperkalemia, acidosis, pulmonary edema, and acute renal failure. As in other organs, ischemic injury in skeletal muscle is initiated during hypoxia and is aggravated by reoxygenation during blood reperfusion. This study aimed at examining the protective effect of antioxidative enzymes, superoxide dismutase and catalase, on ischemia-reperfusion injury in skeletal muscle. Skeletal muscles in rat hindlimb were subjected to in vivo ischemia by clamping both the infrarenal aorta and the bilateral femoral arteries for $4 \mathrm{~h}$ and to the subsequent blood reperfusion for $1 \mathrm{~h}$ under profound anesthesia with pentobarbitone. An attempt was made to suppress the muscle injury by infusing superoxide dismutase and catalase $5 \mathrm{~min}$ before starting reperfusion. Tissue water content was significantly increased by reperfusion. The increase in the water content was suppressed by the addition of those scavengers. Plasma levels of creatine phosphokinase and lactate dehy-
\end{abstract}

\footnotetext{
*To whom correspondence should be addressed.

${ }^{a}$ A.Y. was supported by a grant from the Japanese Foundation of Cardiovascular Research, Tokyo. The present study was also supported by a general grant; and H.T., by a Grant-in-Aid (C) from the Ministry of Education, Science, Sports and Culture of Japan. ${ }^{\text {b Present address: }}$ First Department of Biochemistry, Kyorin University School of Medicine, Mitaka, Tokyo

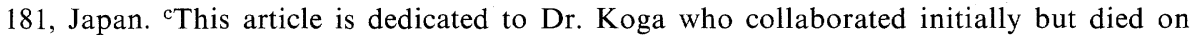
Nov., 4th, 1995. ${ }^{\text {d}}$ To whom correspondence and reprint requests should be addressed at Department of Hygiene, Miyazaki Medical College, 5200 Kiwara, Kiyotake-cho, Miyazakigun, Miyazaki 889-16, Japan. FAX: +81-985-85-5177.

Abbreviations used: CAT, catalase; CCO, cytochrome $c$ oxidase; CPK, creatine phosphokinase; LDH, lactate dehydrogenase; MDA, malondialdehyde; OPR, oxidative phosphorylation rate; $\mathrm{P} / \mathrm{O}$, phosphate-to-oxygen ratio; $\mathrm{RCI}$, respiratory control index; SOD, superoxide dismutase; TBA, thiobarbituric acid; TEP, 2,2,2'2'-tetraethoxypropane.
} 
drogenase were increased by ischemia and reperfusion. Addition of the scavengers did not suppress these increases, indicating that scavengers were not effective in suppressing the cellular injury, which might have begun during the ischemia and which might have been exacerbated by the reperfusion. Mitochondrial oxidative phosphorylation was significantly suppressed by ischemia and reperfusion. Such scavengers restored the inhibited mitochondrial function. These results indicate that the radical scavengers tested were effective to protect mitochondrial functions against ischemia-reperfusion insults. Although the present study was carried out on an animal model, administration of these radical scavengers may be effective to reduce injury due to acute arterial occlusion and crush syndrome.

Key Words: ischemia-reperfusion, skeletal muscle, superoxide dismutase, catalase, mitochondria

Prolonged skeletal muscle ischemia often induces progressive edema and necrosis, and leads to serious structural and functional muscle injury, even when blood flow is restarted [1]. Serious cases are accompanied by potentially lifethreatening systemic complications, such as hypotension, hyperkalemia, acidosis, pulmonary edema and acute renal failure $[2,3]$. Such pathologic consequences have been reported in acute arterial occlusion and crush syndrome, which was prevalent in the aftermath of the devastating earthquake that occurred in 1995 in the Kobe area, Japan. As in other organs, ischemic injury in skeletal muscle is initiated during hypoxia and is aggravated by reoxygenation during blood reperfusion, viz., ischemia-reperfusion injury. The aim of the present study was to analyze the effect of ischemia and reperfusion on mitochondrial function in skeletal muscle and to examine the possible protective effects of the antioxidative enzymes, superoxide dismutase (SOD) and catalase (CAT), on the mitochondrial injury. For this study, we used an acute arterial occlusion model in the rat. A variety of models have been developed to study ischemia-reperfusion injury in skeletal muscle [4-7], and free radicals are thought to play a key role in the pathogenesis of injury as in other organs such as brain, heart, kidney, and liver. Possible sources of free radical generators are the xanthine-xanthine oxidase system, leukocytes, and the mitochondrial electron transfer chain $[1,8]$. There have been many reports describing the efficacy of various radical scavengers on the reduction of ischemia-reperfusion injury $[1,8]$. A decrease in mitochondrial oxidative phosphorylation has been blamed for ischemia-reperfusion injury [916]. However, few reports have examined in detail the relationship between the ischemia-reperfusion injury and the mitochondrial function in skeletal muscle. Since skeletal muscle depends on the glycolytic pathway for its energy supply [17], it is not so vulnerable to ischemia-reperfusion injury as other organs. It has been 
indicated that mitochondria play an important role in the supply of energy current 'ATP' for the restoration of intracellular ionic homeostasis [18]. It is, therefore, likely that preserving and hastening recovery of mitochondrial respiratory function is necessary for reducing ischemic-reperfusion injury and for restoration of the physiological function of skeletal muscle. The present study shows that the administration of both SOD and CAT suppresses (i) tissue edema induced by reperfusion and (ii) inhibition of mitochondrial function caused by ischemia and reperfusion. However, in this protocol, SOD and CAT, administered immediately before the reperfusion, failed to suppress the elevation of plasma levels of creatine phosphokinase and lactate dehydrogenase, indicating that myocytolysis was initiated during ischemia and exacerbated the reperfusion.

\section{MATERIALS AND METHODS}

Animals. We followed our college's regulations for animal research, which were based on the Guide for the Care and Use of Laboratory Animals prepared by the Institute of Laboratory Animal Resources and published by the National Institutes of Health (NIH Publication No. 85-23, 1985). Specific pathogen-free (SPF) Wistar rats (male, 13-14 weeks old, Kiwa Co., Wakayama, Japan) were kept at the Experimental Animal Center of Miyazaki Medical College under SPF conditions and fed a conventional diet and water ad libitum for at least 2 weeks.

Model for acute arterial occlusion. All procedures for the rats were carried out at $22-25^{\circ} \mathrm{C}$ and approximately $70 \%$ relative humidity. Profound anesthesia was introduced and maintained by intraperitoneal injection of sodium pentobarbitone $(50 \mathrm{mg} / \mathrm{kg})$. Animals were allowed to breath spontaneously. The left external jugular vein was exposed and a cannula (24 gauge) was set in place. After intravenous heparinization $(400 \mathrm{IU} / \mathrm{kg})$, physiological saline was continuously infused with a pump at $3 \mathrm{ml} / \mathrm{kg} / \mathrm{h}$ through the cannula. After upper median laparotomy and bilateral inguinal incisions had been made, the infrarenal aorta and the bilateral femoral arteries were exposed.

Experimental groups. Rats were randomly assigned to four groups: control, ischemic, ischemic-reperfused, and the enzyme groups (Fig. 1). The control group received a sham operation, in which the abdominal and inguinal wounds were closed with nylon sutures after exposing both the aorta and the bilateral femoral arteries. After $1 \mathrm{~h}$, laparotomy was again carried out and rats were sacrificed by collecting whole blood from the suprarenal aorta. Plantaris and gastrocnemius muscle tissues were immediately removed from the right hindlimb for mitochondrial preparation, since those were distinguished from macroscopic observation according to Armstrong and Phelps [19]. The ischemic group received clamping at both the infrarenal aorta and the bilateral femoral arteries with atraumatic vascular clips (TKL-1 for the aorta and TKS-1 for the femoral arteries, Kyowa Precision Instruments Co., Tokyo). The time between anesthesia and clamping was $30 \mathrm{~min}$. After clamping, each incision was closed by suturing. After $4 \mathrm{~h}$ of is- 


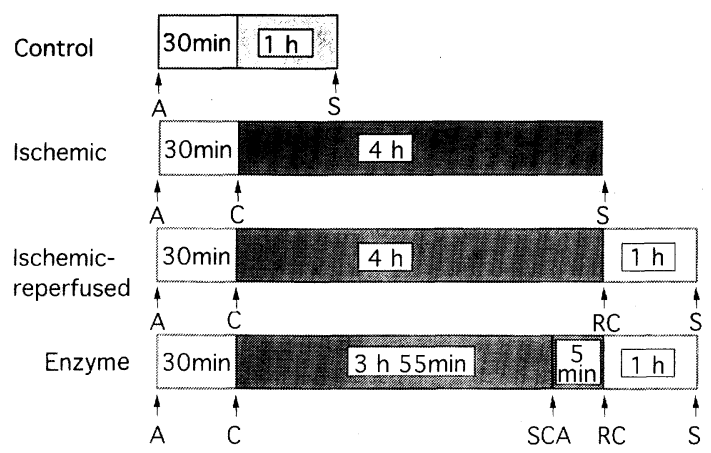

Fig. 1. Experimental protocol. After anesthesia (A) and heparinization, upper median laparotomy and bilateral inguinal incisions were performed, and the infrarenal aorta and bilateral femoral arteries were exposed. Control group received a sham operation. After $1 \mathrm{~h}$, rats were sacrificed (S) by collecting whole blood. Plantaris and gastrocnemius were immediately removed from the hindlimb for mitochondrial preparation. Ischemicgroup animals were clamped $(\mathrm{C})$ at the infrarenal aorta and bilateral femoral arteries with vascular clips. We set the time between anesthesia and clamping at $30 \mathrm{~min}$. After $4 \mathrm{~h}$ of ischemia, blood and muscle were sampled as in the control group. In the ischemic-reperfused group, clamps were removed after $4 \mathrm{~h}$ of ischemia (RC) and blood was reperfused for $1 \mathrm{~h}$. Rats were sacrificed and muscles were obtained as in the control group. The enzyme group received superoxide dismutase (SOD) and catalase (CAT) 5 min before removal of clamps. Procedures to isolate blood and muscle were similar in all groups.

chemia, sampling of blood and muscles was carried out as in the control group. The ischemic-reperfused group received $1 \mathrm{~h}$ of blood reperfusion after $4 \mathrm{~h}$ of ischemia. Rats were sacrificed and muscles were obtained as in the control group. The enzyme group received SOD $(20,000 \mathrm{IU} / \mathrm{kg})$ and CAT $(20,000 \mathrm{IU} / \mathrm{kg})$ dissolved in $0.3 \mathrm{ml}$ of physiological saline from the cannula at $5 \mathrm{~min}$ before the removal of clamps and were then reperfused for the $1 \mathrm{~h}$. Procedures for isolating blood and muscles were similar in all other groups.

Estimation of tissue water content. About $200 \mathrm{mg}$ wet weight of a muscular specimen at the central portion of both muscles was dried at $80^{\circ} \mathrm{C}$ for $24 \mathrm{~h}$. Tissue water content (\%) was calculated as (wet weight-dry weight)/wet weight $\times 100$.

Blood samples. Blood specimens drawn from abdominal aorta were centrifuged at $3,000 \times g$ for $20 \mathrm{~min}$ at room temperature and the plasma thus obtained was frozen at $-80^{\circ} \mathrm{C}$ until examined.

Isolation of mitochondrial fraction. All isolation procedures were carried out at $2-4^{\circ} \mathrm{C}$, and mitochondrial fractions were prepared from both plantaris and gastrocnemius tissue from the right hindlimb according to the method of Chappel and Perry [20] with a slight modification. After dissecting vessels, connective tissues, and fascia, each muscle specimen was finely diced with scissors, washed once, and homogenized in $10 \mathrm{vol}$ of an ice-cold solution containing $0.1 \mathrm{M} \mathrm{KCl}$, $1 \mathrm{~mm}$ ATP, $5 \mathrm{~mm} \mathrm{MgCl}_{2}, 1 \mathrm{~mm} \mathrm{O}, \mathrm{O}^{\prime}$-bis(2-aminoethyl) ethyleneglycol-N,N,N', 
$\mathrm{N}^{\prime}$-tetraacetic acid (EGTA), $50 \mathrm{~mm}$ 2-[4-(2-hydroxyethyl)-1-piperazinyl] ethanesulfonic acid (Hepes) ( $\mathrm{pH}$ 7.4) with the aid of a Polytron homogenizer (Brinkmann Instruments Co., NY) at the half maximal setting for $20 \mathrm{~s}$ (10-s on, 10-s off, 10-s on). The homogenate was centrifuged at $600 \times g$ for $10 \mathrm{~min}$ and the supernatant was passed through 8 layers of medical gauze. The filtrate was centrifuged at $600 \times$ $g$ for $10 \mathrm{~min}$, and the supernatant was filtered and centrifuged at $10,000 \times g$ for 10 min. The resultant pellet was suspended in the isolation buffer described above and centrifuged at $10,000 \times g$ for $10 \mathrm{~min}$. The surface of the pellet was rinsed with $0.25 \mathrm{M}$ sucrose, and the pellet was resuspended in $0.5 \mathrm{ml}$ of a solution containing $0.25 \mathrm{M}$ sucrose, $1 \mathrm{~mm} \mathrm{~N}, \mathrm{~N}, \mathrm{~N}^{\prime}, \mathrm{N}^{\prime}$-ethylenediamine tetraacetic acid (EDTA) and 20 $\mathrm{mM}$ Hepes ( $\mathrm{pH} 7.4)$ as the crude mitochondrial fraction. Mitochondrial respiratory function and cytochrome $c$ oxidase activity were determined immediately after the isolation. A portion of the suspension was kept for determination of lipid peroxide level after addition of $1 / 20 \mathrm{vol}$ of $0.05 \%$ butylated hydroxytoluene in dimethylsulfoxide and kept in liquid $\mathrm{N}_{2}$ until examined. Gravitational force in centrifugation was set at the middle point of tubes throughout the experiment.

Mitochondrial respiratory function. Respiratory function of mitochondria in the presence of succinate as a substrate was determined by measuring oxygen consumption with an oxygen probe (Yellow Springs Instrument, Co., Inc., Yellow Springs, $\mathrm{OH})$ at $25^{\circ} \mathrm{C}$. The reaction was started by the addition of $25 \mu 1$ of

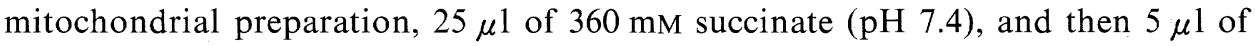
$26.1 \mathrm{mM}$ ADP ( $\mathrm{pH} 7.4$ ) to $1.85 \mathrm{ml}$ of a medium containing $0.25 \mathrm{M}$ sucrose, $20 \mathrm{~mm}$ $\mathrm{KCl}, 5 \mathrm{mM} \mathrm{MgCl} 2,10 \mathrm{~mm}$ potassium phosphate buffer ( $\mathrm{pH} 7.4$ ), and $20 \mathrm{~mm}$ Hepes ( $\mathrm{pH} 7.4$ ), which had been equilibrated with air for at least $30 \mathrm{~min}$ at $25^{\circ} \mathrm{C}$. The initial oxygen concentration in the medium was assumed to be 450 nanoatom $/ \mathrm{ml}$. Respiratory control index (RCI) was calculated by dividing the rate of oxygen consumption after addition of ADP (state 3 ) by the rate before addition of ADP (state 4$)$. Phosphate-to-oxygen ratio $(\mathrm{P} / \mathrm{O})$ was calculated by dividing the amount of consumed ADP by the amount of oxygen consumed in the state 3 condition. Oxidative phosphorylation rate (OPR) was calculated by multiplying the state 3 rate by the P/O. ATP synthesis rate, which was equal to OPR per g wet tissue, was the product of OPR and mitochondrial yield.

Assay of cytochrome $c$ oxidase (CCO) activity. Activity of $\mathrm{CCO}$ was determined from the initial rate of oxidation of reduced cytochrome $c$ by spectrophotometric titration at $550 \mathrm{~nm}$ in $0.1 \%$ Tween 80 and $0.1 \mathrm{M}$ potassium phosphate buffer $\left(\mathrm{pH} \mathrm{7.0)}\right.$ at $37^{\circ} \mathrm{C}$. The differential molar extinction coefficient of reduced minus oxidized cytochrome $c$ was assumed to be $19.6 \mathrm{~mm}^{-1} \cdot \mathrm{cm}^{-1}$ [21].

Determination of lipid peroxide level in mitochondria. Lipid peroxide level in the mitochondria was estimated according to the method of Yagi [22] with a slight modification. The modification made was that the mitochondrial suspension $(50 \mu 1)$ was mixed with $50 \mu 1$ of $8.1 \%$ (w/v) sodium dodecylsulfate, followed by the addition of $0.75 \mathrm{ml}$ of distilled water and $0.5 \mathrm{ml}$ of $20 \%(\mathrm{w} / \mathrm{v})$ sodium acetate $(\mathrm{pH}$ 3.5). Precipitation of mitochondria with silicotungstic acid prior to treatment with 
sodium dodecylsulfate was done to remove the sucrose that could interfere with color development in the following procedure. After mixing the solubilized mitochondria with $0.5 \mathrm{ml}$ of $0.8 \%(\mathrm{w} / \mathrm{v})$ thiobarbituric acid (TBA), the mixture was heated at $95^{\circ} \mathrm{C}$ for $1 \mathrm{~h}$ and was immediately chilled with tap water. Tetraethoxypropane (TEP) was used as a standard to estimate the MDA formed in samples, and lipid peroxide level was expressed in terms of nmol $\mathrm{MDA} / \mathrm{mg}$ protein. Samples were diluted with physiologic saline to give the absorbance below 0.3 at the time of measurement.

Assay of plasma creatine phosphokinase (CPK) and lactate dehydrogenase (LDH) activities. Activity of CPK was determined by titration of initial rate of reduction of $2 \mathrm{mM} \mathrm{NADP}^{+}$in the presence of $30 \mathrm{~mm}$ creatine phosphate, $2 \mathrm{~mm}$ ADP, $20 \mathrm{~mm}$ glucose, $2.5 \mathrm{IU} / \mathrm{ml}$ hexokinase, $2.5 \mathrm{IU} / \mathrm{ml}$ glucose-6-phosphate dehydrogenase, $20 \mathrm{~mm} \mathrm{~N}$-acetylcysteine, $10 \mathrm{~mm}$ magnesium acetate, $5 \mathrm{mM}$ AMP, $30 \mathrm{~mm}$ $\mathrm{P}^{1}, \mathrm{P}^{5}$-(diadenosine- $\left.5^{\prime}\right)$ pentaphosphate $\left(\mathrm{Ap}_{5} \mathrm{~A}\right)$, and $100 \mathrm{mM}$ imidazole buffer $(\mathrm{pH}$ 6.7) at $37^{\circ} \mathrm{C}$. Concentration of NADPH was estimated by assuming that the millimolar extinction coefficient at $340 \mathrm{~nm}$ was 6.22 .

In a parallel experiment, we confirmed by separation with isoelectric focusing (Special Gel 8, Corning, NY) that the CPK isoenzyme in plasma was mainly CPK-MM. Electrophoresis to analyze CPK isoenzymes was carried out with $1 \mu 1$ of the sample in $50 \mathrm{~mm}$ 2-hydroxy-3-morpholinopropanesulfonic acid (Mops) buffer ( $\mathrm{pH} \mathrm{7.8)}$ at $90 \mathrm{~V}$ for $20 \mathrm{~min}$. Fluorescence of NADH was measured in the presence of $6 \mathrm{mM} \mathrm{NAD}^{+}, 90 \mathrm{~mm}$ creatine phosphate, $12 \mathrm{mM}$ ADP, $60 \mathrm{~mm}$ glucose, $9 \mathrm{IU} / \mathrm{ml}$ hexokinase, $7.5 \mathrm{IU} / \mathrm{ml}$ glucose-6-phosphate dehydrogenase, $60 \mathrm{mM} \mathrm{N}$-acetylcysteine, $60 \mathrm{mM}$ magnesium acetate, $15 \mathrm{~mm}$ AMP, $10 \mathrm{~mm} \mathrm{Ap}$ A, and $47 \mathrm{~mm} 2$ morpholinoethanesulfonate buffer $\left(\mathrm{pH} \mathrm{6.7)}\right.$ at $37^{\circ} \mathrm{C}$ for $20 \mathrm{~min}$. Relative intensity of fluorescence of NADH was determined, and isoenzyme composition was estimated from distribution of fluorescence intensity, by using a Corning 780 Fluorometer/Densitometer (Ciba Corning, NY).

Activity of LDH was assayed by titration of initial drop in the absorption of $0.21 \mathrm{~mm} \mathrm{NADH}$ in the presence of $0.77 \mathrm{~mm}$ sodium pyruvate and $48 \mathrm{~mm}$ sodium phosphate buffer $\left(\mathrm{pH} \mathrm{7.5)}\right.$ at $37^{\circ} \mathrm{C}$. Concentration of $\mathrm{NADH}$ was estimated by assuming that the millimolar extinction coefficient at $340 \mathrm{~nm}$ was 6.22 .

Others. Protein concentration was determined by the method of Lowry et al. [23] with bovine serum albumin used as a standard. Concentration of ADP was determined spectrophotometrically (Cary 2290, Victoria, Australia) at $259 \mathrm{~nm}$ with $15.4 \mathrm{mM}^{-1} \cdot \mathrm{cm}^{-1}$ as the extinction coefficient.

Reagents. Sodium pentobarbitone was obtained from Abbot Laboratories (IL); heparin sulfate, from NOVA Industry A/S (Bagsvaerd, Denmark); cytochrome $c$, from Sigma Chemical Co. (MO); SOD, from Toyobo Biochemicals (Osaka, Japan); CAT, from Calbiochem (CA); and ADP from Kohjin Chemicals (Tokyo, Japan). Hepes and EDTA were from Dojindo Laboratories (Kumamoto, Japan). All other reagents used were of analytical grade and purchased from either Wako Pure Chemicals (Osaka, Japan) or Nacalai Tesque (Kyoto, Japan). 
Statistic treatment. All data were expressed as mean \pm standard error of means (SEM). Significant difference within each group was referred to analysis of variance (ANOVA) followed by Scheffe's F-test. Difference between ischemicreperfused and scavenged groups was analyzed by Student's $t$-test. Differences were considered significant when $p$ was $<0.05$.

\section{RESULTS}

\section{Tissue water content}

Figure 2 compares the tissue water content among the control, ischemic, ischemic-reperfused, and enzyme groups. The tissue water content was not increased by ischemia, whereas reperfusion significantly increased it. The content after addition of the enzymes was apparently higher than that in the ischemic group, though the difference was not statistically significant. On the other hand,

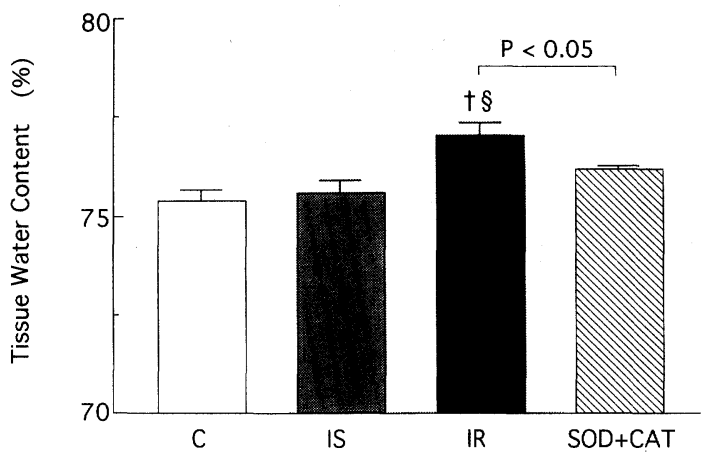

Fig. 2. Tissue water content of control $(\mathrm{C}, n=8)$, ischemic (IS, $n=7)$, ischemic-reperfused (IR, $n=8)$ and enzyme (SOD +CAT, $n=7$ ) groups. Tissue water content (\%) was calculated by dividing (wet weight - dry weight) by dry weight $\times 100$. All data are expressed as the mean \pm SEM. Significance of difference among the groups was determined by analysis of variance (ANOVA): $\dagger$, vs. the control $(p<0.01)$; $\S$, vs. the ischemic $(p<0.01)$. Difference between the ischemic-reperfused and the enzyme groups was determined by Student's $t$-test $(p<0.05)$.

Table 1. Yield of mitochondrial protein.

\begin{tabular}{lcc}
\hline Group & Number & $\begin{array}{c}\text { Yield } \\
\text { (mg protein/g dry weight) }\end{array}$ \\
\hline Control & 10 & $7.785 \pm 0.359$ \\
Ischemic & 9 & $7.372 \pm 0.452$ \\
Ischemic-reperfused & 10 & $7.712 \pm 0.552$ \\
Enzyme & 8 & $7.778 \pm 0.513$ \\
\hline
\end{tabular}

Values are expressed as the mean \pm SEM. Significance of differences among the groups was determined by analysis of variance (ANOVA) and between ischemic-reperfused and enzyme groups by Student's $t$-test. There was no significant difference among the groups.

Vol. 23, No. 1, 1997 
addition of the enzymes significantly reduced the water content compared with the content of the ischemic-reperfused group, indicating that the enzymes were effective in suppressing the tissue edema induced by reperfusion.
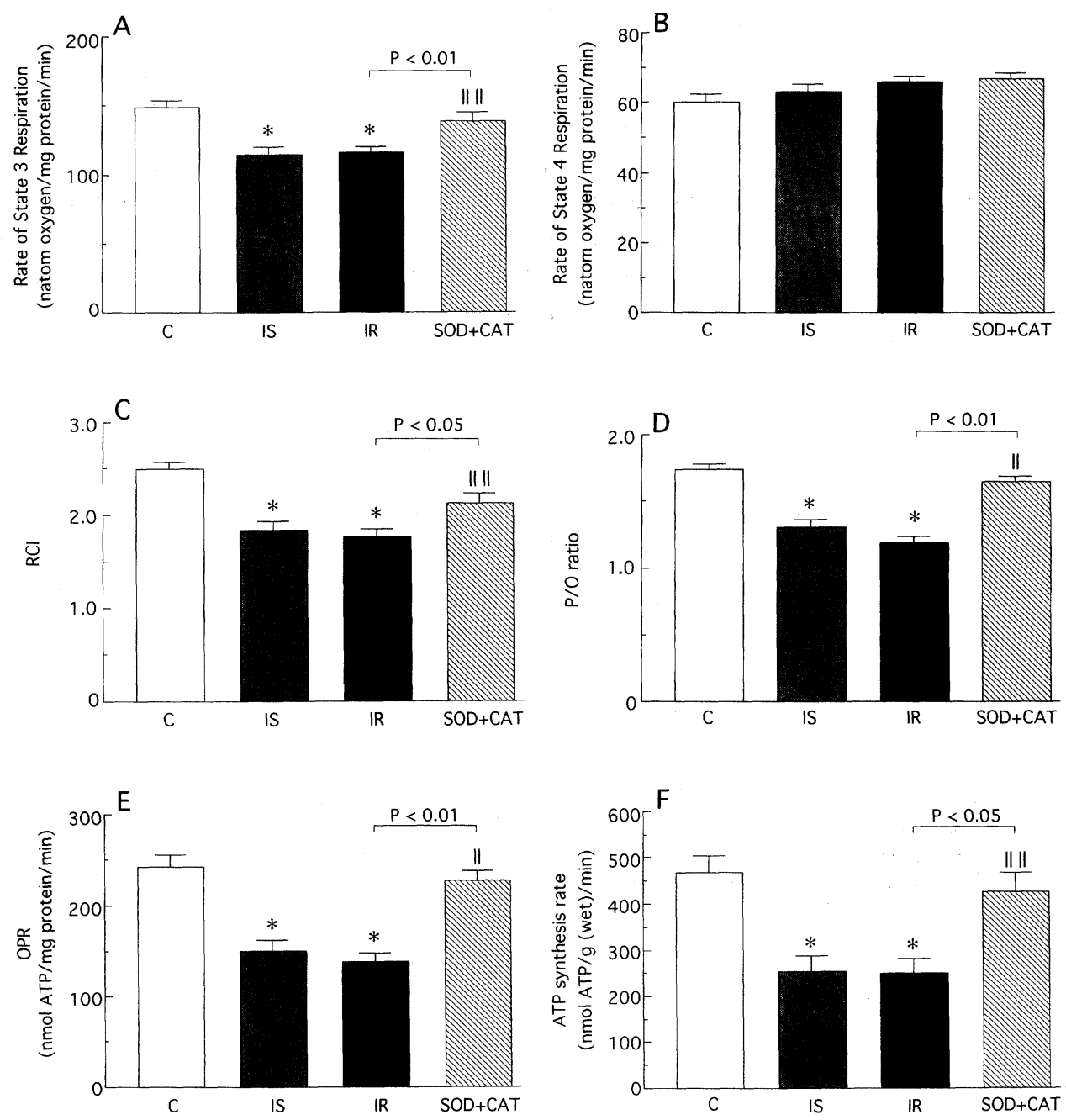

Fig. 3. Respiratory function of mitochondria isolated from control $(\mathrm{C}, n=10)$, ischemic (IS, $n=9$ ), ischemic-reperfused (IR, $n=10$ ), and enzyme (SOD +CAT, $n=8$ ) groups. (A) state 3 rate; (B) state 4 rate; (C) respiratory control index (RCI); (D) phosphate-tooxygen ratio $(\mathrm{P} / \mathrm{O})$; (E) oxidative phosphorylation rate (OPR); and (F) ATP synthesis rate. All data are expressed as the mean \pm SEM. Significance of differences among the groups was determined by analysis of variance (ANOVA): $*$, vs. the control $(p<0.01)$; $\|(p<0.01)$ and \|\|$(p<0.05)$, vs. the ischemic. Difference between the ischemicreperfused and the enzyme groups was determined by Student's $t$-test, and significant differences are shown in the figure. There was no significant difference in the state 4 rate. 
Table 2. Activity of cytochrome $c$ oxidase.

\begin{tabular}{lcc}
\hline Group & Number & $\begin{array}{c}\text { CCO activity } \\
(\mu \mathrm{mol} / \mathrm{mg} \cdot \mathrm{min})\end{array}$ \\
\hline Control & 10 & $410.0 \pm 27.85$ \\
Ischemic & 9 & $357.2 \pm 44.31$ \\
Ischemic-reperfused & 10 & $353.3 \pm 32.25$ \\
Enzyme & 8 & $362.4 \pm 26.72$ \\
\hline
\end{tabular}

Cytochrome $c$ oxidase (CCO) activities are expressed as the mean \pm SEM. Significance of differences among the groups was determined by analysis of variance (ANOVA) and between the ischemic-reperfused and the enzyme groups by Student's $t$-test. There was no significant difference among the groups.

\section{Mitochondrial yield and respiration and cytochrome $c$ oxidase activity}

Mitochondrial yields showed no significant variance among the groups, although the ischemic group showed apparently a lower yield than the other three groups (Table 1). State 3 respiration was slowed by ischemia, though no further decrease was induced by reperfusion (Fig. 3A). Addition of the enzymes restored the suppressed activity almost to the control value. This resulted in a significantly high activity in the scavenged group compared with that in the ischemic-reperfused group. State 4 respiration rate was slightly increased by ischemia and reperfusion, but the increase was not statistically significant (Fig. 3B). RCI values were significantly lessened by ischemia and reperfusion, as seen in state 3 respiration (Fig. 3C). Addition of the enzymes significantly increased RCI values compared with the ischemic and ischemic-reperfused levels. Ischemia and reperfusion significantly lowered the $\mathrm{P} / \mathrm{O}$ ratio, whereas the enzymes restored the value to the control level (Fig. 3D). Reperfusion apparently lowered the $\mathrm{P} / \mathrm{O}$ after ischemia, though the difference was not statistically significant. OPR showed changes similar to those in the $\mathrm{P} / \mathrm{O}$ value (Fig. 3E). As expected from no marked difference in mitochondrial yield, changes in ATP synthesis rate coincided with those in $\mathrm{P} / \mathrm{O}$ values (Fig. 3F).

Cytochrome $c$ oxidase activity was apparently higher in the control than in the other three groups, though the difference was not significant (Table 2).

\section{Lipid peroxide formation in mitochondrial fraction}

Figure 4 compares the lipid peroxide levels in mitochondrial fractions isolated from the four experimental groups. The lipid peroxide level was apparently increased by ischemia, though the increase was not statistically significant. On the other hand, ischemia-reperfusion significantly increased the lipid peroxide level compared with the control group value. Addition of SOD and CAT significantly suppressed the increase in lipid peroxides that resulted from ischemia-reperfusion.

\section{Plasma CPK and LDH activities}

Figure 5 shows changes in plasma levels of CPK and LDH, which are often referred to as indicators of cellular disintegration of muscle [7]. Levels of both Vol. 23, No. 1, 1997 


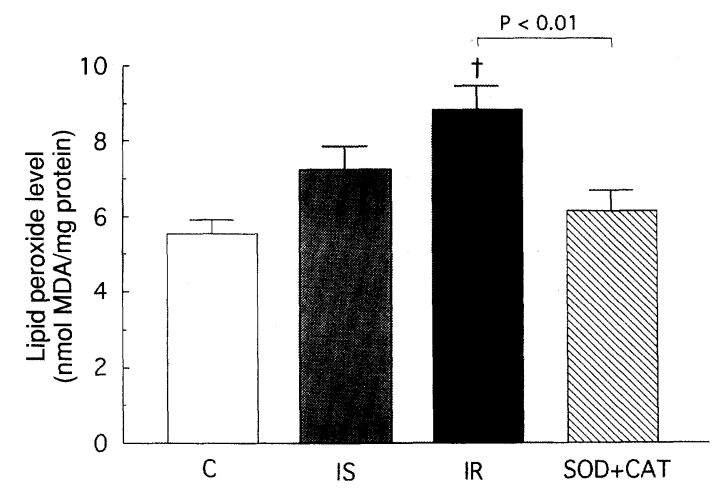

Fig. 4. Lipid peroxide level in mitochondrial fraction from the control $(\mathrm{C}, n=10)$, ischemic (IS, $n=9$ ), ischemic-reperfused (IR, $n=10$ ), and enzyme (SOD +CAT, $n=8$ ) groups. All data are expressed as the mean \pm SEM. Significant differences among the groups were determined by analysis of variance (ANOVA): $\dagger$, vs. the control $(p<0.01)$. Difference between the ischemic-reperfused and the enzyme groups was determined by Student's $t$-test $(p<0.01)$.
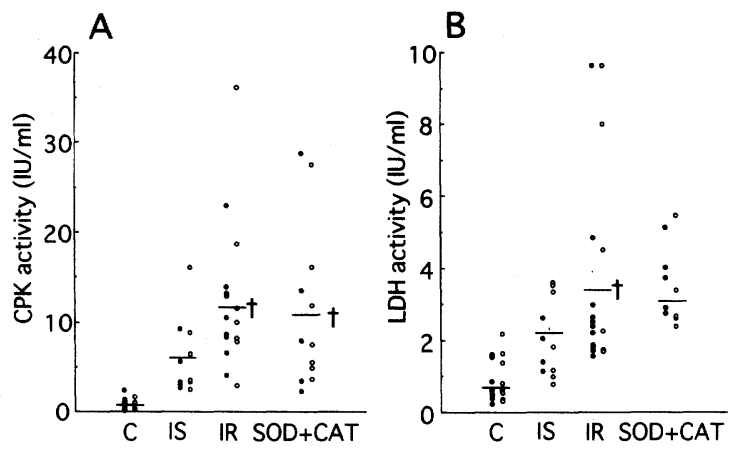

Fig. 5. Plasma levels of CPK (A) and LDH (B) in the control (C), ischemic (IS), ischemicreperfused (IR), and enzyme (SOD $+\mathrm{CAT}$ ) groups. Rats selected for mitochondrial isolation are indicated as open circles, and others, as closed circles. It should be noted that open and closed circles are equally scattered, indicating that no intentional selection was made to collect data for both parameters. Bars indicate mean values. Significant differences amog the groups were determined by analysis of variance (ANOVA): $\dagger$, vs. the control $(p<0.01)$. Difference between the ischemic-reperfused and the enzyme groups was not significant, as determined by Student's $t$-test.

enzymes were greatly scattered in all groups. We indicated individual rats used for measurement of both mitochondrial function and plasma levels of CPK and LDH by the open circles in Fig. 5. Both CPK and LDH levels were apparently higher in the ischemic group than in the control group, though the difference was not significant. CPK levels were higher in both the reperfused and the enzyme groups than those in the control group, whereas the $\mathrm{LDH}$ levels were found to be 
significantly elevated by reperfusion alone. There was no significant difference between the ischemic-reperfused group and the enzyme one for either enzyme.

\section{DISCUSSION}

Extensive studies have been conducted to analyze the etiology of ischemiareperfusion injury in a variety of organs [8]. However, studies of ischemia-reperfusion injury of skeletal muscle have been limited due to difficulty in establishing an in vivo model to ensure complete ischemia, since the vascular system in skeletal muscles is very complex, i.e., presence of collateral flow, compared with that in brain, heart, kidney, and liver. Attempts have been made to study ischemiareperfusion injury of skeletal muscle in isolated canine gracilis [4], rat hindlimb tied with a tourniquet [7], rat hindlimb after aortic clamping [6], and amputated rat hindlimb [5]. However, these models have met criticism regarding incomplete ischemia, tissue deterioration, obstruction of venous blood flow, disturbance of nervous system, and destruction of fascia [4]. In the present study, we rendered rat hindlimb muscle ischemic by clamping both the infrarenal aorta and the bilateral femoral arteries for $4 \mathrm{~h}$ with atraumatic vascular clips. Although complete ischemia was not attained, neither obstruction of venous flow nor nervous system disturbances were observed; but fascia was maintained, making this a good model to mimic clinical cases of acute arterial occlusion. Yokota et al. [6] reported, using microsphere distribution analysis, that blood flow after clamping the infrarenal aorta was reduced by $83.4 \%$. However, since we found that bleeding occurred when the femoral arteries were dissected, even after clamping the infrarenal aorta, we clamped the bilateral femoral arteries to reduce the blood flow into the sample muscles. Therefore, blood flow into muscles would be lower than that reported by Yokota et al. [6], although we did not estimate the exact volume of blood flow in the present study.

The purpose of the present study was to observe whether SOD and CAT were capable of protecting skeletal muscle from ischemia-reperfusion injury at the tissue and cellular level. Our aim was to evaluate the clinical relevance of radical scavengers for acute arterial occlusion and crush syndrome. We assessed the injury at the tissue level both by estimating the extent of leakage of cellular enzymes, $\mathrm{CPK}$ and $\mathrm{LDH}$, into the bloodstream and by evaluating tissue edema as tissue water content in the presence and absence of SOD and CAT. We also measured activities of the mitochondrial respiratory system that produces ATP for cellular homeostatic reactions.

Various procedures have been used to reduce ischemia-reperfusion injury of skeletal muscle: reperfusion with radical scavengers $[1,6,8]$, with hypoxic perfusate [1], with leukocyte-depleted blood $[1,6,24]$, with controlled pressure $[1,5]$ or under hypothermia [5]. Scavengers and antioxidative enzymes tested include allopurinol, dimethylsulfoxide, SOD and CAT, and time of administration varied among reports. Hydroperoxide, which SOD generates during quench-

Vol. 23, No. 1, 1997 
ing of superoxide anions, is the substrate for more cytotoxic hydroxyl radicals in the presence of metal catalysts (Fenton's reaction). A few reports described that single use of SOD aggravated ischemia-reperfusion injury [1]. Since skeletal muscle has an abundant supply of ferrous/ferric ions, such as iron in myoglobin molecules, we supplemented the SOD with CAT as a scavenger for $\mathrm{H}_{2} \mathrm{O}_{2}$. Ischemia-reperfusion injury often develops prior to surgical treatment, and in clinical cases such as acute arterial occlusion, pretreatment to reduce the injury is not possible. Furthermore, it has been shown that ischemia-reperfusion injury is induced in the early phase of the reperfusion process [25], and SOD is known to have a short half-life in the bloodstream [1]. Therefore, we administered both SOD and CAT 5 min before the removal of clamps to evaluate the efficacy of these enzymes in clinical use.

Since CPK and LDH are known as cytosolic enzymes that leak into the bloodstream when cells are injured, they are often used as markers for tissue injury of skeletal muscle [7]. Administration of SOD and CAT did not suppress the elevated activities in plasma after ischemia and reperfusion treatments, but it lessened leakage of both enzymes only slightly. This fact may be explained if the cellular structure started to deteriorate during the ischemic period and if the reperfusion released both enzymes into the bloodstream. On the other hand, administration of SOD and CAT suppressed the increase in tissue water content after the reperfusion. It is known that increases both in the capillary pressure and in the permeability across the vascular bed, which result in increased compartment pressure, also aggravate the reperfusion injury [26]. Therefore, it is likely that SOD and CAT suppressed some of these increases. These results suggest that administration of SOD and CAT was effective in suppressing the tissue edema, of which reperfusion is the primary cause, although SOD and CAT were not effective in reducing the injuries that had occurred during the preceding ischemic period.

Armstrong and Phelps [19] estimated the absolute and relative masses of the three types of skeletal muscle fibers in the hindlimb of the male Sprague-Dawley rat. Accordingly, the skeletal muscle fibers analyzed in the present study were about $6 \%$ type-I fiber and $94 \%$ type-II fiber. Skeletal muscle has been indicated to be tolerant of ischemia because of low dependency on aerobic respiration [17]. Kuzon et al. [4] reported that the canine gracilis muscle sustained normal tissue ATP and glycogen levels for 4 and $6 \mathrm{~h}$ of ischemia, respectively, whereas they found that creatine phosphate levels started to decrease $2 \mathrm{~h}$ after the onset of ischemia and were restored by reperfusion. On the other hand, lactate levels increased after ischemia and were restored after reperfusion, whereas glucose-6phosphate levels gradually increased during both ischemia and reperfusion [6]. It was accordingly concluded that ATP levels were maintained by activated glycolysis. These reports indicated that a decrease in oxygen supply shifted energy producing system towards glycolysis, while ATP synthesis by mitochondrial oxidative phosphorylation was suppressed.

Few reports have described the mitochondrial role in ischemia-reperfusion 
processes by examining the function of mitochondria isolated from ischemiareperfusion treated muscle. The production of ATP by the mitochondria is likely to be important for the restoration of homeostasis of muscle cells after an ischemiareperfusion insult. Therefore, reduction of ischemia-reperfusion injuries and maintenance of muscular function are likely to demand preserved mitochondrial function. Mitochondrial yield did not vary among the three groups, indicating that our isolation procedures were reproducible and that comparison of mitochondrial function among the groups was feasible. Furthermore, the rat groups were equally sampled for preparation of both mitochondria and plasma as shown in Fig. 5, indicating that mitochondrial damage and leakage of cytosolic enzymes were directly comparable in the present study. We added $1 \mathrm{mM}$ ATP during the mitochondrial isolation procedure to dissociate actomyosin in order to facilitate isolation of subsarcolemmal and interfibrillar mitochondria. It was less likely that the concentration of contaminating ATP in the reaction mixture affected the measurement of oxidative phosphorylation activity, since the highest possible amount of ADP, even if all ATP had been hydrolyzed, was 5 nmol, significantly lower than the ADP added $(130.5 \mathrm{nmol})$. Several reports have shown that ischemia-reperfusion inhibited mitochondrial oxidative phosphorylation in heart $[9,10]$ and liver $[11]$. We used succinate as the substrate to follow the changes in oxidative phosphorylation capability through the complex II, since the complex I has been reported to be extremely vulnerable to ischemia-reperfusion [12]. State 3, RCI, P/O ratio, OPR, and ATP synthesis rate were all lowered by $4 \mathrm{~h}$ of ischemia and showed no recovery after reperfusion. Cytochrome $c$ oxidase activity showed no significant change after ischemia-reperfusion, which is consistent with other reports [13]. These facts suggest that inhibition of the state 3 respiration during ischemia-reperfusion of skeletal muscle might occur before complex IV.

Free radicals $[10,14]$, accumulation of free fatty acids [15], and accumulation of $\mathrm{Ca}^{2+}[16]$ have been blamed for ischemia-reperfusion injuries of mitochondria, although the detailed mechanisms have not been well clarified. We estimated lipid peroxidation of mitochondrial membranes and found that the lipid peroxide level was significantly elevated by reperfusion. This elevation was markedly suppressed by addition of both SOD and CAT. The xanthine-xanthine oxidase system and mitochondrial respiratory chain have been listed as intracellular sources of free radicals during ischemia-reperfusion processes $[1,8]$, whereas activated neutrophils and macrophages are believed to be extracellular sources of active oxygen species [27]. Participation of infiltrating neutrophils, especially in skeletal muscle, has been predicted to play a major role in ischemia-reperfusion injury $[6,24]$. However, microscopic observation of muscle specimens showed no apparent infiltration of neutrophils into muscles in the present study (figure not shown). It is not likely that the large SOD and CAT molecules crossed the sarcolemma and penetrated into the intracellular space to directly inhibit the oxidation of mitochondrial membranes. Therefore, inhibition of lipid peroxide formation by SOD and CAT may be explained if SOD and CAT scavenged active 
oxygen produced by activated leukocytes in the blood and/or if extracellular SOD and CAT contributed to lessen the rate of exhaustion of intracellular radical scavengers such as glutathione to scavenge active oxygen species that were generated during ischemia-reperfusion processes.

We have established an acute arterial occlusion model of rat hindlimb skeletal muscles by clamping the infrarenal aorta and bilateral femoral arteries to study the effects of in vivo ischemia-reperfusion on cellular integrity and mitochondrial function. Mitochondrial respiration was lowered by both $4 \mathrm{~h}$ of ischemia alone and ischemia with $1 \mathrm{~h}$ of reperfusion. These two treatment groups also showed an increase in lipid peroxide levels. Addition of SOD and CAT at $5 \mathrm{~min}$ before onset of reperfusion did not reverse tissue damage, although the lipid peroxide levels were significantly suppressed. These results obtained suggest that the addition of SOD and CAT was not effective in reducing physical disintegration of muscle cells during the ischemic period. However, these enzymes markedly protected the muscle from tissue edema and mitochondrial dysfunction after ischemia-reperfusion, which could eventually support restoration of cellular activities. We therefore expect that administration of SOD and CAT via the bloodstream before reperfusion will prove clinically useful to reduce muscular injury in acute arterial occlusion and also crush syndrome.

We thank Drs. H. Maruyama of the Second Department of Internal Medicine and K. Nakamura of the Second Department of Surgery, Miyazaki Medical College, for their valuable suggestions. We also thank Ms. Catherine C. Bryson, BA/BS, for reading the manuscript.

\section{REFERENCES}

1. Akimitsu, T., Gute, D.C., Jerome, S.N., and Korthuis, R.J. (1994): Reactive oxygen metabolites and their consequences, in Ischemia-Reperfusion Injury of Skeletal Muscle, ed. by Fantini, G.A., RG Landes Co., Austin, TX, Chapter 2, pp. 5-31.

2. Davis, J.M., and Fantini, G.A. (1994): Remote effects: Lung injury, in Ischemia-Reperfusion Injury of Skeletal Muscle, ed. by Fantini, G.A., RG Landes Co., Austin, TX, Chapter 9, pp. 5-31.

3. Himovici, H. (1979): Metabolic complications of acute arterial occlusions. J. Cardiovasc. Surg. Torino, 20, 349-357.

4. Kuzon, W.M., Jr., Walker, P.M., Mickle, D.A.G., Harris, K.A., Pynn, B.R., and Mckee, N.H. (1986): An isolated skeletal muscle model suitable for acute ischemia studies. J. Surg. Res., 41, 24-32.

5. Beyersdorf, F., Matheis, G., Krüger, S., Hanselmann, A., Freisleben, H.-G., Zimmer, G., and Satter, P. (1989): Avoiding reperfusion injury after limb revascularization: Experimental observations and recommendations for clinical application. J. Vasc. Surg., 9, 757-766.

6. Yokota, J., Minei, J.P., Fantini, G.A., and Shires, G.T. (1989): Role of leukocytes in reperfusion injury of skeletal muscle after partial ischemia. Am. J. Physiol., 257, H10681075.

7. Presta, M., and Ragnotti, G. (1981): Quantification of damage to striated muscle after normothermic or hypothermic ischemia. Clin. Chem., 27, 297-302.

8. Bulkley, G.B. (1987): Free radical-mediated reperfusion injury: A selective review. $B r . J$. Cancer, 55, Suppl., 66-73. 
9. Yano, H., Takenaka, H., Onitsuka, T., Koga, Y., and Hamada, M. (1993): Cardioplegic effect of University of Wisconsin solution on hypothermic ischemia of rat myocardium assessed by mitochondrial oxidative phosphorylation. J. Thorac. Cardiovasc. Surg., 106, 502-510.

10. Blasig, I.E., Bor, P., Tosaki, A., Szekeres, L., and Löwe, H. (1986): Effect of activated oxygen species on mitochondria isolated from myocardium after reperfusion injury. Gen. Physiol. Biophys., 6, 655-658.

11. Gonzales-Flecha, B., Cutrin, J.C., and Boveris, A. (1993): Time course and mechanism of oxidative stress and tissue damage in rat liver subjected to in vivo ischemia-reperfusion. $J$. Clin. Invest., 91, 456-464.

12. Hardy, L., Clark, J.B., Darley-Usmar, V.M., Smith, D.R., and Stone, D. (1991): Reoxygenation-dependent decrease in mitochondrial NADH: CoQ reductase (complex I) activity in the hypoxic/reoxygenated rat heart. Biochem. J., 274, 133-137.

13. Nishida, T., Shibata, H., Koseki, M., Nakao, K., Kawashima, Y., Yoshida, Y., and Tagawa, K. (1987): Peroxidative injury of the mitochondrial respiratory chain during reperfusion of hypothermic rat liver. Biochim. Biophys. Acta, 890, 82-88.

14. Malis, C.D., and Bonventre, J.V. (1986): Mechanism of calcium potentiation of oxygen free radical injury to renal mitochondria. A model for post-ischemic and toxic mitochondrial damage. J. Biol. Chem., 261, 14201-14208.

15. Pearce, F.J., Forster, J., DeLeeuw, G., Williamson, J.R., and Tutwiler, G.F. (1979): Inhibition of fatty acid oxidation in normal and hypoxic perfused rat hearts by 2tetradecylglycidic acid. J. Mol. Cardiol., 11, 893-915.

16. Nayler, W.G., Panagiotopoulos, S., Elz, J.S., and Daly, M.J. (1988): Calcium-mediated damage during post-ischemic reperfusion. J. Mol. Cell. Cardiol., 20, Suppl. 2, 41-54.

17. Haljamäe, H., and Enger, E. (1975): Human skeletal muscle energy metabolism during and after complete tourniquet ischemia. Ann. Surg., 182, 9-14.

18. Benson, D.W., Weinstein, E.S., Niffenegger, J., Yokum, M., and Fry, D.E. (1985): Subpopulations of skeletal muscle mitochondria: Response to ischemia. Am. Surg., 51, 682686.

19. Armstrong, R.B., and Phelps, R.O. (1984): Muscle fiber type composition of the rat hindlimb. Am. J. Anat., 171, 259-272.

20. Chappel, J.B., and Perry, S.J. (1954): Biochemical and osmotic properties of skeletal muscle mitochondria. Nature, 173, 1094-1095.

21. Yonetani, T. (1965): Studies on cytochrome $c$ peroxidase. J. Biol. Chem., 240, 4509-4514.

22. Yagi, K. (1976): A simple fluorometric assay for lipoperoxide in blood plasma. Biochem. Med., 15, 212-216.

23. Lowry, O.H., Rosebrough, N.J., Farr, A.L., and Randall, R.J. (1951): Protein measurement with Folin phenol reagent. J. Biol. Chem., 193, 265-275.

24. Kirschner, R.E., and Fantini, G.A. (1994): Role of neutrophils, in Ischemia-Reperfusion Injury of Skeletal Muscle, ed. by Fantini, G.A., RG Landes Co., Austin TX, Chapter 2, pp. 33-59.

25. Jolly, S.R., Kane, W.J., Bailie, M.B., Abrams, G.D., and Lucchesi, B.R. (1984): Canine myocardial reperfusion injury, its reduction by the combined administration of superoxide dismutase and catalase. Circ. Res., 54, 277-285.

26. Perler, B.A., Tohmeh, A.G., and Bulkley, G.B. (1990): Inhibition of the compartment syndrome by the ablation of free radical-mediated reperfusion injury. Surgery, 108, 40-47.

27. Koppenol, W.H., Moreno, J.J., Pryor, W.A., Ischiropoulos, H., and Beckman, J.S. (1992): Peroxynitrite, a cloaked oxidant formed by nitric oxide and superoxide. Chem. Res. Toxicol., 5, 834-842. 